\title{
Análise de Técnicas Computacionais Usadas na Segmentação de Nódulos Pulmonares
}

\author{
Jorge Paulo Soares Rocha Filho, Paulo Eduardo Ambrósio \\ Programa de pós-graduação em Modelagem Computacional em Ciência e Tecnologia - \\ Universidade Estadual de Santa Cruz (UESC) \\ Ilhéus - BA - Brasil \\ jorgepaulors@gmail.com, peambrosio@uesc.br
}

\begin{abstract}
According to WHO (World Health Organization) lung cancer is the most common cause of death by cancer and your early detection is directly related to the effectiveness of the treatment and with the chances of cure. One of the most used techniques to diagnose lung cancer involves digital image analysis obtained by conventional x-ray and computed tomography. Also image processing has been standing out as one of the research fields in ascension. This work aims the comparative analysis of different digital image processing techniques to identify and validate which of them are most applicable to the use for lung nodules segmentation on computed tomography scans. As its final objective, this work aims to describe and integrate the validated techniques to an open platform to support lung cancer diagnosis.
\end{abstract}

Resumo. O câncer pulmonar é a causa mais comum de mortes por câncer segundo a OMS (Organização Mundial de Saúde) e a sua deteç̧ão precoce está diretamente relacionada com a efetividade do tratamento e com as chances de cura. Uma das principais técnicas diagnósticas para essa doença envolve a análise de imagens digitais obtidas por raio-x convencional $e$ tomografia computadorizada, sendo que o processamento computadorizado dessas imagens tem se destacado como um dos campos de pesquisa em grande ascensão. Este trabalho tem como objetivo a análise comparativa de diferentes técnicas de processamento digital de imagens, buscando identificar e validar quais delas são mais indicadas para a segmentação de nódulos pulmonares em imagens de tomografia computadorizada. Como objetivo final do projeto, o conjunto de técnicas validadas terão seus respectivos algoritmos descritos e integrados em plataforma aberta de apoio ao diagnóstico de câncer de pulmão.

\section{Introdução}

O diagnóstico do câncer pulmonar não deve ser feito somente utilizando um tipo de exame, pois cada um deles possui vantagens e grandes desvantagens. Os exames mais utilizados para a detecção das lesões são o raio-x da região do tórax e a tomografia computadorizada do pulmão, mas outros exames são muito importantes, justamente, por desvantagens que os exames principais apresentam. $\mathrm{O}$ raio-x é visto ainda como a 
"chave" para a detecção de câncer de pulmão. Esse exame tem alta sensibilidade para tumores periféricos e baixo custo. A tomografia computadorizada é muito precisa, proporcionando informações de tamanho, localização e níveis de invasão do tumor. [Uehara et al 1998] Nesse contexto, verifica-se importantes e promissores estudos mais aprofundados com a tomografia computadorizada para que o processo de diagnóstico seja cada vez mais automatizado e simplificado e seja adquirida maior confiabilidade do resultado apresentado nesse exame. Espera-se que futuramente o exame seja suficiente para o diagnóstico e um processo automático para a análise das imagens da tomografia seria crucial para acelerar esse diagnóstico e, em cascata, aumentar as possibilidades de cura.

\section{Segmentação de Imagens}

As técnicas de segmentação de imagens utilizadas neste trabalho são técnicas de crescimento de regiões e de contorno ativo (snake).

Abordagens por contorno ativo fazem uso efetivo de informações prévias específicas dos objetos e isso torna os algoritmos intrínsecamente eficientes. Além disso, algoritmos de contorno ativo são aplicados em regiões específicas da imagem ao invés de serem aplicados na imagem inteira, o que faz a sua eficiência ser ainda maior.

As técnicas de crescimento de regiões consistem basicamente em agrupar pixels ou semi-regiões em regiões maiores baseados em determinada característica [Gonzalez et al 2009]. Com as tomografias computadorizadas, serão utilizados os níveis de cinza, pois as imagens são monocromáticas e os níveis de cinza são a característica disponível para caracterizar uma região.

\section{Materiais e Métodos}

Para o desenvolvimento deste trabalho foi utilizada a base de imagens disponibilizada pelo Lung Image Database Consortium e Image Database Resource Initiative (LIDCIDRI) [Armato et al 2011]. A linguagem de programação utilizada para a implementação dos algoritmos de segmentação de imagens por crescimento de regiões e contorno ativo é o MATLAB.

As imagens tomográficas disponíveis na base LIDC-IDRI contam com a anotação de diagnóstico e segmentação manual de nódulos pulmonares realizada por especialistas. Para o desenvolvimento do trabalho foram selecionadas as imagens com indicação confirmada de nódulos, feita por especialistas, visando a adequada análise e validação das técnicas propostas e posterior comparação e integração em ambiente de apoio ao diagnóstico [Ferreira-Junior et al 2016].

Foram implementadas duas variações de contorno ativo e duas variações de crescimento de regiões. Essas quatro técnicas estão sendo aplicadas nas imagens previamente selecionadas para que a fase de comparações e análise das técnicas seja iniciada.

\section{Resultados Parciais e Esperados}

As quatro técnicas escolhidas para a análise e implementadas no MATLAB foram aplicadas em algumas imagens, previamente selecionadas para o estudo inicial, gerando 
então as áreas segmentadas com o auxílio dos dados disponibilizados na base de imagens.

Na Figura 1 está o exemplo de uma das tomografias computadorizadas onde um nódulo foi identificado por especialistas e na Figura 2 estão os resultados das segmentações feitas por cada um dos métodos citados.

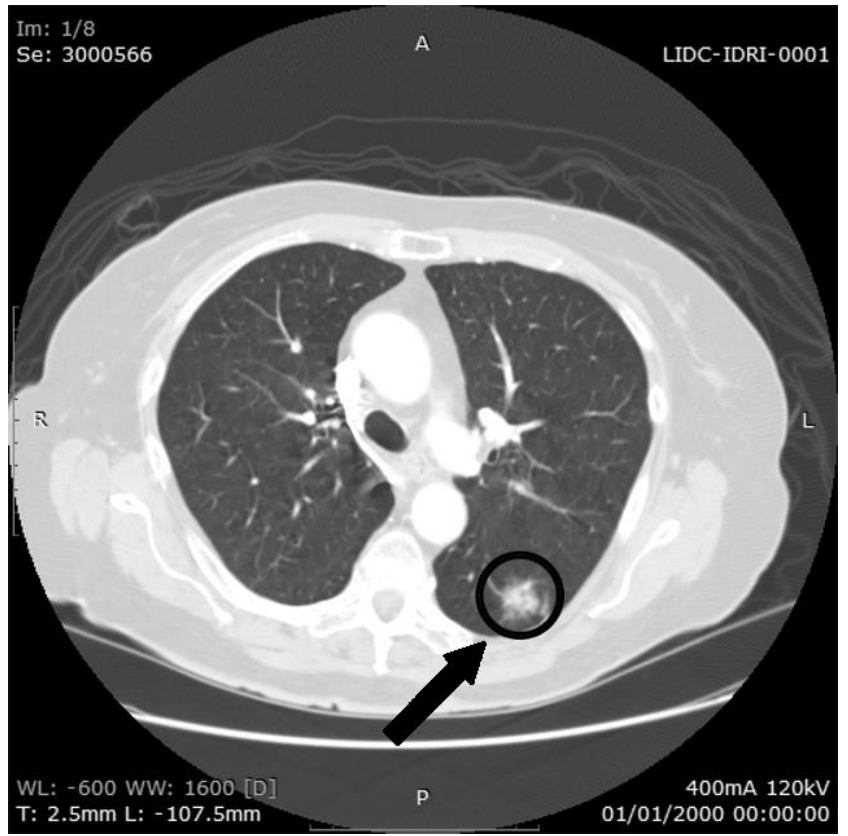

Figura 1. Imagem original de uma tomografia computadorizada que contém nódulo identificado por especialistas

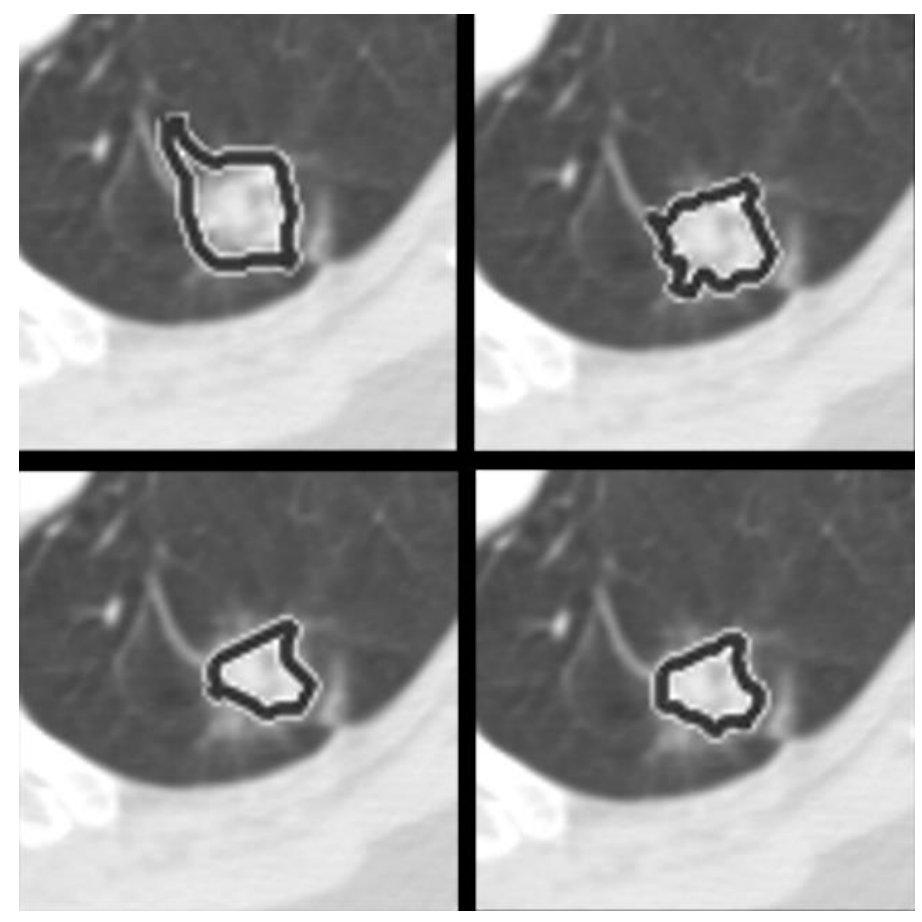

Figura 2. Resultados das segmentações por contorno ativo (superior esquerdo e superior direito) e por crescimento de regiões (inferior esquerdo e inferior direito). 
Atualmente estão sendo realizadas as implementações dos métodos escolhidos para comparar e analisar as técnicas computacionais estudadas, em relação à segmentação feita manualmente pelos especialistas. As características das segmentações a serem comparadas são área e perímetro e além dessas duas características, será utilizada a informação do centróide da massa para que seja feita uma comparação por sobreposição das imagens.

Assim que todos os métodos de comparação tiverem sido implementados, será selecionado o grupo de imagens no qual as técnicas de segmentação serão aplicadas e, em seguida, devidamente analisadas atingindo os resultados finais esperados nesse trabalho.

\section{Referências}

Uehara, C.; Jamnik, S. \& Santoro, I.L. (1998) "Câncer de pulmão". Medicina: Ribeirão Preto, 31:266-276

Gonzalez, R.C. and Woods, R.E. (2009) Processamento de imagens digitais. Pearson.

Armato SG III, McLennan G, Bidaut L, McNitt-Gray MF, Meyer CR, Reeves AP, Zhao B, Aberle DR, Henschke CI, Hoffman EA, Kazerooni EA, MacMahon H, van Beek EJR, Yankelevitz D, et al. (2011) "The Lung Image Database Consortium (LIDC) and Image Database Resource Initiative (IDRI): A completed reference database of lung nodules on CT scans". Medical Physics, 38: 915-931.

Ferreira-Junior, J.R., Oliveira, M.C. \& Azevedo-Marques, P.M. (2016) "Cloud-based NoSQL open database of pulmonary nodules for computer-aided lung cancer diagnosis and reproducible research". Journal of Digital Imaging, 29:716-729. 